Itinéraires Itinéraires

Littérature, textes, cultures

\title{
Une énonciation sans communication : les tatouages scripturaux
}

\author{
Marie-Anne Paveau
}

\section{(2) OpenEdition}

Journals

Édition électronique

URL : http://journals.openedition.org/itineraires/353

DOI : 10.4000/itineraires.353

ISSN : 2427-920X

Éditeur

Pléiade

\section{Édition imprimée}

Date de publication : 1 mai 2009

Pagination : 81-105

ISBN : 978-2-296-08444-5

ISSN : $2100-1340$

\section{Référence électronique}

Marie-Anne Paveau, « Une énonciation sans communication : les tatouages scripturaux », Itinéraires [En ligne], 2009-1 | 2009, mis en ligne le 03 septembre 2014, consulté le 01 juillet 2020. URL : http:// journals.openedition.org/itineraires/353; DOI : https://doi.org/10.4000/itineraires.353

Ce document a été généré automatiquement le 1 juillet 2020

\section{cc)}

Itinéraires est mis à disposition selon les termes de la licence Creative Commons Attribution - Pas d'Utilisation Commerciale - Pas de Modification 4.0 International. 


\title{
Une énonciation sans communication : les tatouages scripturaux
}

\author{
Marie-Anne Paveau
}

\section{NOTE DE L'AUTEUR}

Cet article adopte les rectifications orthographiques proposées dans le Journal officiel du 6 décembre 1990.

Le renouvellement des corps d'exemples n'est pas toujours dû à des renouvellements théoriques (il peut y avoir des raisons purement idéologiques qui tiennent au contenu qu'expriment aussi les

exemples). Mais les changements théoriques profonds s'accompagnent souvent $\mathrm{du}$ changement des exemples. Cela se voit particulièrement lorsqu'il y a une extension $\mathrm{du}$

champ des phénomènes que l'on prend en compte.

S. Auroux, La Raison, le langage et les normes Mon tatouage sur le poignet, c'est un symbole

bouddhiste, je l'ai fait pour moi, c'est pour pouvoir le regarder quand je dessine et que je fais de la calligraphie. Celui sur la poitrine, c'est pour pouvoir le voir dans le miroir. Jean-François, vendeur chez Mona Lisait, juillet 2008 


\section{Introduction}

Les tatouages ont été jusqu'à présent, en sciences humaines et sociales, des objets pour l'anthropologie ou la philosophie (Le Breton 1992, 2002, 2003, Andrieu 2003, 2004a et b), l'archéologie (Renaut 2004), la psychanalyse (Anzieu 1985, Tenenhaus 1995) ou la médecine (Lacassagne 1881, Gilbert 1970, Tagnaouti 1979), mais n'ont pas encore été examinés d'un point de vue discursif ou textuel, que l'approche soit littéraire, linguistique, graphique ou sémiotique. On commence tout juste à considérer les tatouages (écrits ou dessins) comme des écritures corporelles, des graphes cutanés qui constituent à la fois un discours du corps et un discours sur le corps, ce qui est entendu dans ce numéro par le terme corpographèse (Paveau 2006, 2007a). Parler d'écriture implique en principe non seulement la production d'énoncés, mais aussi leur réception et leur lecture, donc un circuit complet de production langagière doté de tous les paramètres habituels de l'énonciation, même s'ils ne sont pas "classiques»: le tatouage écrit fait en effet du corps un support de discours dont tout porte à croire qu'il possède, comme tout discours, un producteur, un récepteur, une forme et une interprétation. Mais la nature du support et des contenus tatoués brouille considérablement le circuit habituel de la production discursive, ce qui rend l'énonciation tatouée extrêmement spécifique, pour deux raisons.

2 D'abord, elle redistribue les cartes de la communication langagière, puisqu'elle opère un désancrage énonciatif de la paire locuteur-interlocuteur et des paramètres spatial et temporel même dans une version renouvelée de la théorie énonciative (comme l'approche en termes de co-énonciation), qu'elle est mobile et éventuellement clandestine (tatouages cachés) et qu'elle mobilise un contexte plus large que celui de la simple interaction.

3 Ensuite, et corrélativement à ce qui précède, l'énonciation tatouée interroge la théorie de l'énonciation elle-même, dans deux de ses fondements: tout d'abord le sujet de l'énonciation qui, bien loin d'être l'instance benvenistienne individuelle et saisissable de la théorie standard, se constitue plutôt de son anonymat, de sa division et des diffractions de ses inscriptions; et ensuite le récepteur ou énonciataire, censé " recevoir » l'énoncé et en construire le sens par cette réception même.

4 Je proposerai d'abord une typologie des corpographies, du mot au texte, constituant une première description de l'écrit tatoué à partir d'un corpus hétérogène et international ( $\mathrm{xIX}^{\mathrm{e}}$ et $\mathrm{xx}^{\mathrm{e}}$ siècles, tatouages européens et américains $\left.{ }^{2}\right)$. Quelques précisions sur ce corpus: en l'état actuel de cette proto-recherche sur les écrits corporels, il est encore exploratoire et a été constitué par sondages : j'ai examiné, dans la littérature publiée sur les tatouages entre la fin $d u x x^{e}$ siècle, période où l'intérêt pour le phénomène est apparu, en particulier à partir des réflexions de Lacassagne et Lombroso sur les marques des criminels, et la fin $d u x^{e}$, où la pratique du tatouage s'est démocratisée, le maximum de publications illustrées intégrant des tatouages scripturaux ou mixtes (verbo-iconiques), mentionnés dans le texte ou présentés sur des photos ou dessins. J'ai également exploré, à partir d'un moteur de recherche, les sites Internet actuellement consacrés aux tatouages ainsi que les banques d'images en ligne, et j'y ai sélectionné, quand elles existaient, les pages de photographies dévolues aux tatouages écrits. De cette recherche documentaire est sorti un ensemble empirique de 300 items environ, à partir duquel j'ai établi une typologie sommaire qui sert de base à la «petite grammaire» qui suit; cette dernière permet de construire un corpus sur 
l'ensemble empirique, puisqu'elle fournit des observables linguistiques. Je me pencherai ensuite sur les pratiques énonciatives dont relèvent les tatouages écrits, et sur la manière dont elles font bouger la théorie standard de renonciation, ce qui me mènera à une réflexion sur le contexte de la production langagière, que des phénomènes comme le tatouage obligent à repenser en termes d'environnement cognitif.

\section{Petite grammaire de l'écriture corporelle}

Les écritures corporelles, ou écrits sur le corps selon des techniques variées (tatoués, scarifiés, inscrits par tonsure, etc.) correspondent à un marquage à la fois social, culturel, religieux, politique et personnel du corps. Elles appartiennent à l'ensemble des inscriptions corporelles, ou corpographies, qu'il s'agisse de dessins tatoués, définitifs ou éphémères, de motifs réalisés par tonsure sur le crâne, de soustractions rituelles corporelles (comme la circoncision), de marquages de la chair par incision ou scarification, ou de modifications de la forme du corps (allongement du crâne, engraissage, amaigrissement, etc.) $)^{3}$. Pour D. Le Breton, les inscriptions corporelles redoublent le statut social des individus sur un mode lisible, en particulier dans les sociétés primitives, à travers leurs trois grandes fonctions : séduction, affiliation et séparation. Dans les sociétés modernes subsistent des formes atténuées de ces marquages, dont les tatouages, qui prennent cependant une ampleur remarquable depuis une dizaine d'années: phénomène de mode, certes, mais aussi, j'y reviendrai, discours contemporain sur le corps (Andrieu 2004b et ici même).

6 Les tatouages écrits sont des productions ambigües, à la fois écriture du collectif sur l'individu (cas des tatouages rituels ou des marquages forcés), et écriture de l'individu par/sur lui-même, le tout dans un rapport complexe du soi à ses extérieurs environnementaux.

7 Avant d'examiner de plus près ce grimoire énonciatif, je propose une typologie formelle des tatouages écrits tels qu'ils apparaissent dans les échantillons qui constituent mon corpus (voir la liste en fin d'article). Ils ont quelques caractéristiques communes, ce qui en fait une catégorie assez stable sur le plan linguistique :

- ce sont souvent des écrits plurisémiotiques, les énoncés étant articulés avec des images (et réciproquement);

- ils sont parfaitement lisibles, le mode graphique choisi, pourtant manuscrit, étant en effet plus celui de la calligraphie (comme sur un monument ou un produit manufacturé) que de l'écriture cursive (comme sur une feuille ou un carnet);

- sauf exceptions notables sur lesquelles je reviendrai, ce sont des formes brèves, qui tiennent sur un espace tégumentaire réduit.

\subsection{Initiales, sigles, chiffres}

Ce sont les formes les plus fréquentes et les plus connues, parfois très codifiées : dans la tradition carcérale, on trouve souvent MA $V$ (mort aux vaches, qui existe aussi sous la forme des trois points en triangle), MAT (mort aux tantes), $V G$ (vengeance) ou encore $B A A$ (bonjour aux amis). A. Lacassagne relève en 1881 sur des prisonniers Mort aux BBT (aux bêtes brutes) et, accompagnée d'un plateau, de deux verres et d'une bouteille, l'inscription VLEBV (vive l'amour et le bon vin). PLV (pour la vie) accompagné d'un cœur 
percé est un tatouage amoureux fréquent. Le corpus de tatouages d'adolescents d'H. Tenenhaus (1993) propose également: TDS (toutes des salopes), NS (né pour souffrir), EDM (enfant de malheur), PCQJ (pour ceux que j'aime) RAM (reste avec moi) ou FLM (fout la merde), qui ne sont pas représentés dans les corpus classiques (Lacassagne, Delarue et Giraud, Pierrat et Guillon). Les initiales d'êtres aimés, perdus ou regrettés, abondent sur toutes les peaux du monde, avec des effets de correction parfois cocasses (telle femme, passée d'un Paul à un Bernard, faisant transformer son $P$ tatoué en $B$ ). Mais la très large diffusion actuelle des tatouages semble ouvrir tous les possibles : sur le site corpsenfolie.fr, on peut voir le sigle ADN (sur le dos) et même un tatouage HIV positif sur le bras. L'initiale comme marquage de propriété, que l'on croirait sortie d'un autre âge, est une pratique certes marginale mais bien réelle, comme le montre un article de Libération de juillet 2008 qui rend compte de l'arrestation de deux proxénètes turco-allemands: "Les frères Baran avaient pour habitude de faire tatouer leurs initiales sur le cou de leurs victimes » (p. 8).

La lettre est également un motif de tatouage en soi : on trouve en effet des formes typographiques, lettres, signes de ponctuation, caractères spéciaux (\& par exemple) ou alphabets entiers (Fig. $1^{4}$ ), avec une inspiration renouvelée par les polices de caractère informatiques (voir Saltz 2006, chapitre 2).

Figure 1

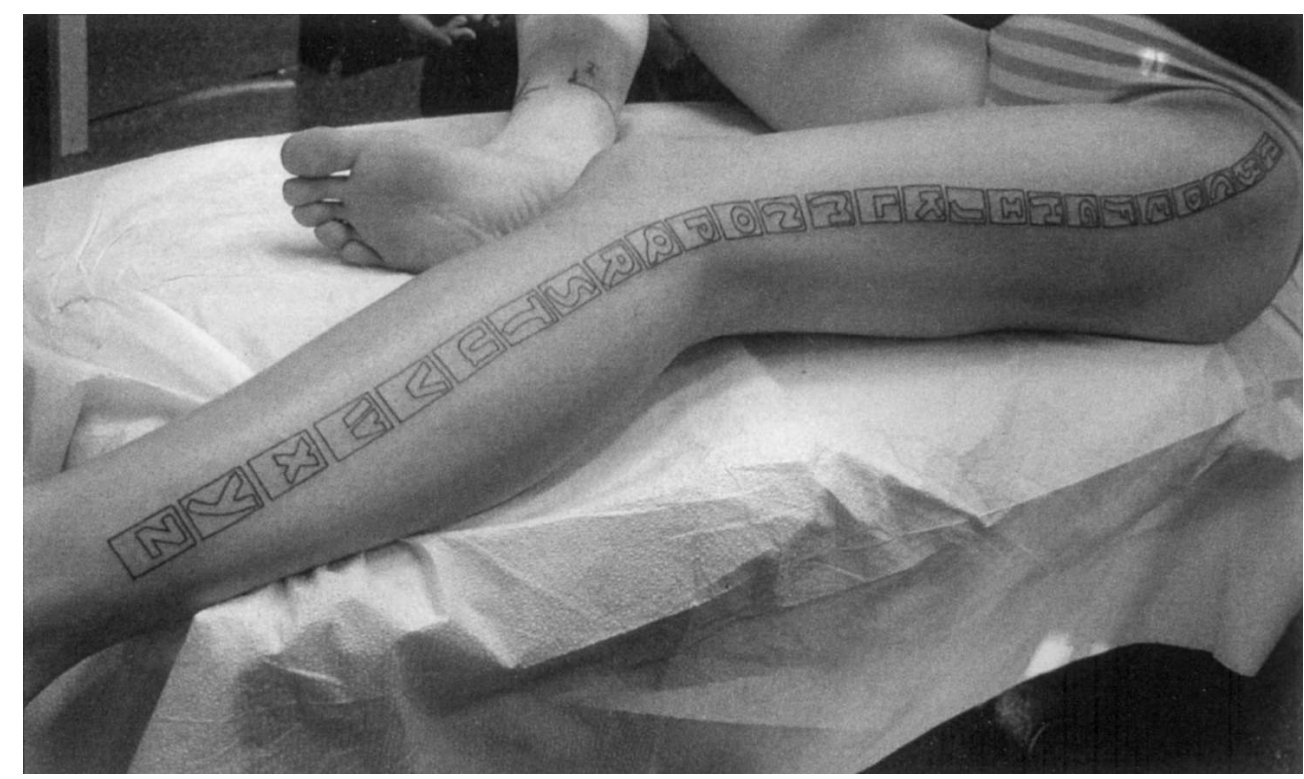

Ina Saltz, Body Types. Intimate Messages Etched in Flesh, New York, HNA, 2006, p. 60.

10 Les chiffres sont essentiellement des dates: dates de naissance, date d'un évènement fondateur dans la vie du tatoué, date d'un évènement historique auquel il se sent relié. On trouve des chiffres porte-bonheur (le 13 en particulier, que l'actrice américaine A. Jolie porte en chiffres romains sur le bras) ou à valeur érotique comme 69. Les tatouages des camps nazis, appliqués à Auschwitz à partir de 1941, sont des immatriculations destinées au tri des humains et à la comptabilité des morts (Baillette 2003, Assouline 2005 ;j'y reviens dans la seconde partie). 


\subsection{Formes brèves, du groupe nominal à la phrase}

11 Les mots isolés constituent une catégorie également fréquente, constituée essentiellement de substantifs non déterminés autour des sentiments ou des rôles familiaux. Les modèles des années 1950 ( «classical tattoo designs ») présentés dans le recueil 1000 Tattoos de H. Schiffmacher (2005) proposent par exemple: Mother, Mom, My boy, (True) Love, Liberty. Les tatouages de criminels relevés par A. Lacassagne et J. Delarue et R. Giraud présentent: Amitié, On trouve passim dans le corpus: Words, Serenity, Recovery, Éclaircissement, Hope, Spes, etc. Les adjectifs sont rares : Insane, Happy. Les noms propres sont également des candidats privilégiés pour les tatouages nominaux, sans doute à cause de leur charge mémorielle et évocatrice forte : noms de pays, de villes (qui peuvent correspondre à des clubs sportifs), noms et prénoms ${ }^{5}$, mais aussi noms de marques, souvent vestimentaires et de préférence sportives (Wrangler, Nike, Adidas).

Se rencontrent également des groupes nominaux (Souvenir d'Afrique), sous forme de phrases averbales (Pas de chance, Sans patrie) ou de constructions prédicatives (Enfant du malheur, Martyr de la liberté) ${ }^{6}$. Les énoncés parémiques, proverbes, cris, devises ou maximes, fournissent une réserve inépuisable d'inscriptions, qu'A. Lacassagne commente ainsi :

Ce sont des sentences, des formules, des proverbes, des dates commémoratives rappelant la date de naissance, de tirage au sort, le numéro de la conscription, le numéro matricule du régiment, la date du tatouage, le jour où il a été condamné. Un homme avait trois inscriptions : c'étaient les dates successives des trois conseils de guerre qui l'avaient condamné. D'autres fois l'inscription semble un cri de colère ou de vengeance: c'est la manifestation évidente d'une nature en révolte contre la société. (Lacassagne $1881: 48$ )

13 Le cri ou la devise est en effet un énoncé de prédilection pour les tatoués; le fonds Lacassagne conservé à la bibliothèque municipale de Lyon contient un ouvrage intitulé Album d'un tatoeur [sic] lyonnais en 1889, constitué d'un recueil de dessins qui fournit une liste de cris :

- Mort aux femmes infidèles

- Mort aux bêtes brutes

- Mort aux tyrans

- Mort aux officiers français

- Mort aux chaouchs

- Mort aux flics

Il faut mentionner également la fameuse devise sur le bras de Bernadotte, Mort aux rois, tatouée pendant la Convention, et qui le met dans un bel embarras le jour où, devenu roi de Suède, malade, il doit découvrir son bras pour une saignée. Précisons, d'après cet exemple en particulier, que la forme devise ou cri est contextuellement déterminée : J. Delarue et R. Giraud rappellent que nombre de poilus se sont fait tatouer des devises patriotiques, antimilitaristes, sentimentales ou amoureuses. Le contexte semble être un élément surdéterminant pour les tatouages écrits. Quelques exemples de maximes tirées de Lacassagne 1881 :

- Vit seul, car les amis sont morts

- La vie n'est que déception

- Plutôt la mort que de changer

- Amis des frères à la côte

- Malheur aux vaincus 
- Mon bras aux amis

- La liberté ou la mort

- Au bout du fossé la culbute

- Haine et mépris aux faux amis

15 Le « dictionnaire analytique » de J. Delarue et R. Giraud (chapitre IV, p. 23-43) propose une liste analogue, quelque 70 ans après :

- Le béguin se paie

- Vers celle qui m'entretiendra

- L'amitié unit les cœurs

- Ami du contraire

- La vie d'un homme

- Justice n'est qu'un vain mot

- Amour trahi demande vengeance

- Eil pour œil, dent pour dent !

- Cent pour cent

- Ni Dieu ni maitre

- Pensez à moi

- Gloire aux femmes!

- Vive l'amour!

- À ma poule, ma femme, ma Gaby, celle que j'aime, etc.

Et les sites Internet et banques d'images permettent d'avoir un aperçu de la maxime moderne tatouée :

- Only God can defeat me (dos, http://gallery.tattoo.com)

- A prayer for the wild at heart, kept in cages (tatouage d'A. Jolie, http://

gallery.tattoo.com)

- Just do it (avec le symbole de la marque Nike, Keith 2007 : 10)

- Straight edge (haut du dos, Keith 2007 : 23)

Sur www.corpsenfolie.com :

- Hasta la muerte (dos)

- Juger sans connaitre (dos)

- Only God can judge me (dos)

- Je l'aime, beaucoup, passionnément, à la folie (pied de femme, surmonté d'une

fleur sur la jambe)

- Vivre libre ou mourir (bras)

- Emprisonné dans la vie / Libre dans l'esprit (bras)

- Main cé Haïtien et main fiè de ça (torse, en créole haïtien, accompagné d'une carte d'Haïti)

17 Le tatouage est souvent verbo-iconique ou verbo-corporel (placé sur une partie du corps qui contribue au sens de l'ensemble). Toujours dans le corpus du «Milieu» on peut relever :

- Tatouages verbo-iconiques

- Ouvre l' [dessin d'un œil] fais gaffe

- Je suis [dessin d'un cochon] au lit

- À la vie, à la mort ! (accompagné de deux mains tenant un poignard)

- Une [dessin d'une pensée] à ma mère ou Mes [pensées dessinées] à ma mère

(J. Delarue et R. Giraud montrent planche 29 un tatoué avec une rangée de plusieurs pensées occupant toute la largeur du dos)

- Comme lui, j'ai souffert (sous un Christ en croix)

- Tatouages verbo-corporels

- À découper selon le pointillé (sur le cou)

- Robinet d'amour (sur le bas-ventre)

- Au bonheur des dames (idem)

- Buvette des sous-offs (idem) 
- Debout là-dedans ! (idem)

- Only for ladies ou Nur für Damen (idem)

- Tout pour toi! (sur la verge)

18 La devise en milieu carcéral ou militaire a pris des formes contemporaines : l'actrice Angelina Jolie porte Know your rights en haut du dos (l'un de ses douze tatouages), et les mots d'ordre politiques constituent l'un des dix chapitres de Body Types, l'ouvrage de I. Saltz sur les tatouages écrits aux États-Unis. Born free, arbore un jeune homme sur chacun des doigts des mains; Equal rights, porte un autre sur l'arrière des bras qui, arrêté par erreur, s'est fait tatouer l'expression pour pouvoir une prochaine fois tourner le dos aux policiers trop zélés; It is our duty to fight for freedom, peut-on lire sur le cou d'un autre.

19 Mais les tatoués ne s'arrêtent pas à la devise et portent des phrases entières, constituant parfois de petits récits. Dans le fonds Lacassagne, on trouve des dessins divers de tatouages et la fiche descriptive des tatouages de Legrand, disciplinaire de 22 ans en 1888. Il porte un tatouage de trois lignes dans la région lombaire : « Le passé m'a trompé, le présent me tourmente et l'avenir m'épouvante » (référence F 61-70). Le docteur Le Blond, qui relève en 1899 des tatouages de prostituées, rapporte des écrits phrastiques et parfois narratifs comme l'indiquent les temps utilisés: une certaine Victorine, «brocheuse de 18 ans ", porte sur le bras gauche j'aime /Alfred georges / pour la vie, avec deux cœurs, deux colombes et des fleurs (p. 40-41); on peut lire sur le bras droit d'une autre j'aime julot pour la vie (p. 52). Une troisième arbore J'aime / mon petit homme / Aimée / P.L.V (puis deux sabres en V), et dessous, A.D., initiales du successeur. La phrase peut se faire petit récit : J'aime [Deux cœurs et colombes] / Charles pronier / Celui qui m'auras apres / Lui seras une tente et une / Coquine (p. 67).

\subsection{Textes littéraires : morceaux choisis}

Enfin ce sont parfois des textes entiers, la plupart du temps littéraires, que certains tatoués réclament. Une tatoueuse chez Dragon Tattoo, rue du Roi-de-Sicile à Paris, me raconte qu'elle vient d'accomplir deux séances de six heures pour écrire sur les bras et le dos d'un homme l'incipit d'un roman de Kafka. Le premier chapitre de l'ouvrage Body Types, intitulé « Literature, Poetry and Lyrics ", montre un extrait de Ulysses de Joyce, le début du monologue d'Hamlet (fig. 2), un passage de L'Enfer de Dante, des citations célèbres de F. Herbert (Fear is the mind killer, dans Dune) ou de J. R. R. Tolkien (Not all who wander are lost), des morceaux de S. Plath, F. O'Hara, W. Stevens, E. Pound, ou même le célèbre excipit du Tractatus logico-philosophicus de L. Wittgenstein (Wovon man nicht sprechen kann, darüber muss man schweigen). On peut aussi rencontrer des prières, tel ce Notre Père en anglais tatoué sur fond de parchemin sur le torse d'un homme en 1950 (Schiffmacher 2005 : 204) ou encore la " Prière de la sérénité » issue du programme en douze étapes des Alcooliques anonymes (Saltz 2006 : 152). Je citerai pour finir le genre particulier de la " poésie du goulag », ainsi présentée par F. Baillette :

Édouard Kouznetsov, qui fut déporté dans les bagnes de Russie, mentionne ainsi l'inscription "sur la partie faciale» (front, menton, joues, cou) de tatouages qualifiés par les autorités disciplinaires de «cyniquement» ou «insolemment antisoviétiques ", pire "à contenu antisoviétique diffamatoire ", comme : Esclave du PCUS, Du pain et la liberté ou encore A bas le Buchenwald des soviets!, Mort aux tyrans et aux tyranneaux! Il se souvient tout particulièrement de l'impertinence d'un petit quatrain tatoué sur la joue d'un compagnon d'infortune :

«Khrouchtchev, je ne le crains pas J'épouserai sa Fourtséva Pour peloter les roberts Les plus 
marxistes de la terre ${ }^{7}$."

(Baillette $2003: 63$ )

Figure 2

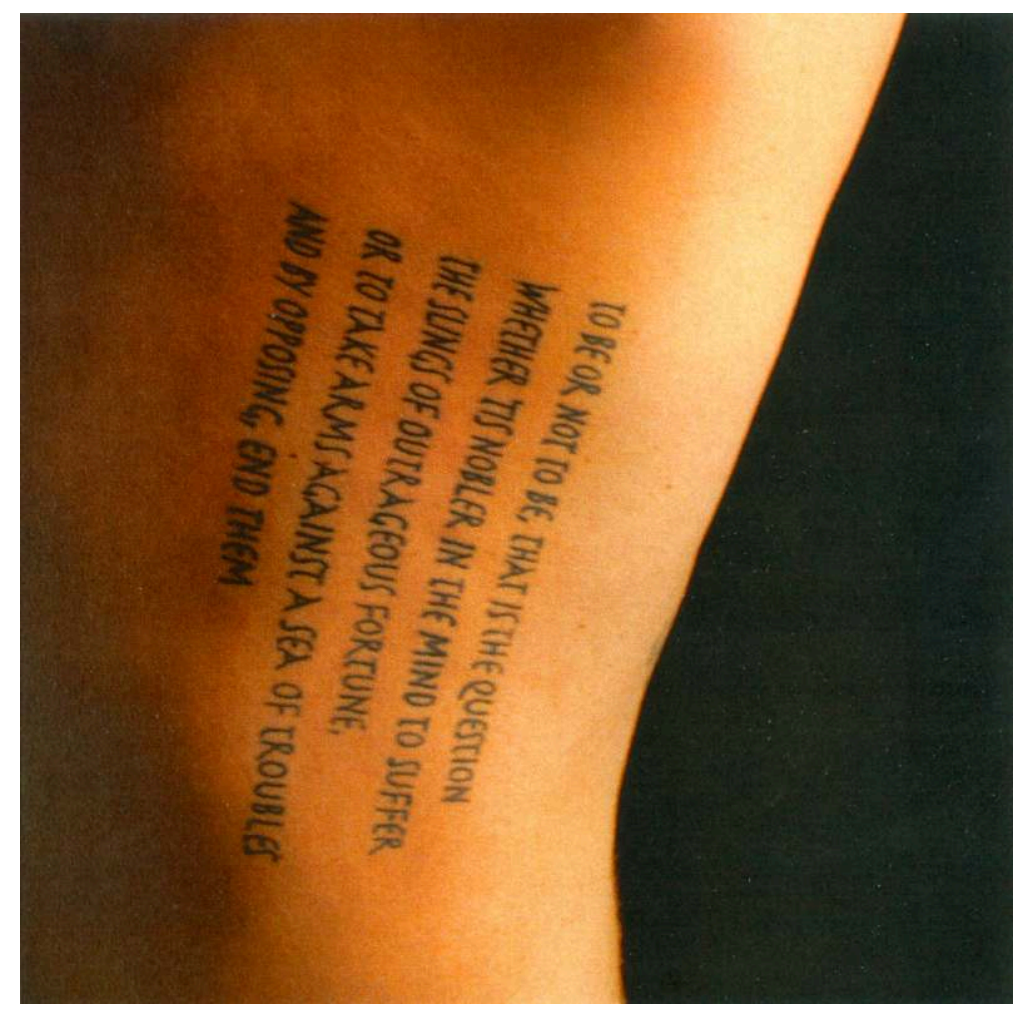

Ina Saltz, Body Types. Intimate Messages Etched in Flesh, New York, HNA, 2006, p. 16.

On le voit, tatoueurs et tatoués exploitent diverses possibilités langagières écrites dans la limite quantitative de l'espace corporel dédié à l'inscription. Les formes énonciatives et scripturales, bien que très inventives, sont limitées car prescrites par le support corporel et le type particulier d'énonciation en cause : pas de texte long, évidemment, très peu d'exclamations, pas de formes dialoguées. Il faut maintenant se demander comment fonctionne, sur le plan énonciatif, cet écrit particulier.

\section{L'énonciation non communicationnelle des écrits tatoués}

Les coordonnées théoriques et épistémologiques de la notion d'énonciation n'ont guère changé depuis les travaux d'É. Benveniste entre 1950 et 1970, même si des modifications parfois importantes ont été apportées aux définitions et aux descriptions des phénomènes énonciatifs. En effet, l'énonciation est toujours envisagée chez la plupart des linguistes à partir d'une situation de communication qui met en jeu des instances locutoires et des paramètres spatio-temporels. C'est la situation princeps mise en place par É. Benveniste dans "Structure des relations de personne dans le verbe " dès 1946, «La nature des pronoms » en 1956 et «L'appareil formel de l'énonciation » en 1970 (Benveniste 1966 et 1974), situation reconduite par des chercheurs comme O. Ducrot et A. Culioli, qui ont pourtant considérablement retravaillé les données de 
départ. Cette conception standard est marquée par deux traits constants, le binarisme et l'anthropocentrisme.

\subsection{Binarisme de la théorie énonciative standard}

23 Les instances énonciatives sont en effet envisagées de manière binaire, un (ou plusieurs) locuteur(s) étant en relation, explicite ou implicite, avec un marquage linguistique en surface (déictiques) ou en profondeur (un énoncé non marqué est réputé marqué en «structure profonde »), avec un (ou plusieurs) interlocuteur(s), dans un cadre dialogal ou polylogal. La notion de coénonciation introduite par F. Jacques (1985) et exploitée linguistiquement par A. Culioli (1990-1999) permet certes de voir les choses de manière plus circulaire que binaire, en proposant un continuum entre les deux agents : les énoncés étant produits à partir des représentations et des réceptions anticipées de l'interlocuteur, l'énonciation devient alors plus une négociation qu'une émission de signes. Mais le cadre binaire locuteur/interlocuteur reste la référence. Les travaux désormais nombreux et devenus canoniques qui s'inspirent du dialogisme de Bakhtine renforcent cette vision, en introduisant du dialogique et donc un ancrage communicationnel dans tous les énoncés, même les plus monologiques et les plus dénués de marques énonciatives. Les tenants de l'indexicalité généralisée des pratiques langagières (pragmaticiens, interactionnistes, en particulier conversationnalistes) maintiennent fermement l'ancrage interactionnel de tout énoncé, même non marqué (voir sur ce point Schegloff 1992 et la synthèse de Kerbrat-Orecchioni 2006). Dans cette perspective, le contexte est construit par les participants à l'interaction, il est en quelque sorte interne à l'interaction, constitué de ce qui leur semble pertinent pour la construction et l'interprétation des énoncés. Il semble donc que la vision doxique soit interactionnelle ou au moins dialogique et que l'idée d'un énoncé dépris de la relation communicationnelle binaire soit hétérodoxe.

Ce sont les travaux sur l'énonciation littéraire qui ont sans doute le plus fortement modifié la théorie standard, en proposant cette alternative hétérodoxe à la «lignée communicationnelle " (Philippe 2000: 4) : l'ouvrage d'A. Banfield, Phrases sans parole, paru en 1982 sous le titre Unspeakable Sentences et traduit en français en 1995, défend en effet l'idée qu'en l'absence de marques énonciatives, il n'y a pas de raison de postuler une situation de communication. Ses analyses concernent au premier chef la figure du narrateur, mais elles sont envisageables à mon sens à propos du lecteur-récepteur. Sur un autre corpus (le discours scientifique), P. Ouellet propose en 1984 la notion de "désénonciation» pour rendre compte du caractère désancré des énoncés scientifiques ${ }^{8}$. La notion est ensuite relayée par celle d' "effacement énonciatif " proposée par R. Vion (Vion 2001) puis retravaillée entre autres par A. Rabatel (par exemple Rabatel 2004). Ces deux positions remettent en cause, chacune à leur manière, le principe d'indexicalité généralisée et permettent de regarder l'énonciation autrement, en dehors du dispositif binaire. C'est la première qui retiendra surtout mon intérêt, la seconde présentant l'absence de marques énonciatives comme une stratégie langagière ou argumentative de surface (donc une sorte de fiction énonciative ${ }^{9}$ ), et ne revenant pas finalement sur l'existence incontestée des deux instances locutoires, énonciateur et énonciataire.

Les paramètres spatio-temporels ont été également retravaillés : on sait désormais que les productions verbales circulent de manière non linéaire et complexe à travers un 
nombre important de filtres contextuels avant d'atteindre leur cible, qui reste cependant irréductiblement et binairement cet autre de l'interlocution. Mais les descriptions du contexte, cet élément nécessaire à toute interprétation des énoncés dans une perspective communicationnelle, sont le plus généralement anthropocentrées, au sens où elles privilégient des données humaines non matérielles comme la culture, les données sociologiques, historiques, bref tout un monde représentationnel qui fait peu de cas des réalités matérielles. Les chercheurs qui travaillent sur la dimension corporelle, en soi, et non comme simple "contexte ", de l'interaction verbale, modifient cette conception en introduisant du matériel biologique rarement sémiotisé, comme le montre ici même $\mathrm{H}$. de Chanay, qui plaide pour un "apport communicatif propre " du corps. Je considère pour ma part que l'ensemble de l'environnement, qu'il soit humain ou non humain, joue un rôle dans les productions verbales, et que le corps bien sûr, mais aussi les objets matériels naturels ou artefactuels, les lieux géographiques et leurs éléments naturels, constituent des contributeurs cognitifs à la production verbale des humains (Paveau 2006, 2007b). De ce fait, l'énonciation apparait comme un phénomène non plus binaire mais distribué, au sens cognitif du terme : l'énoncé circule dans l'environnement à travers des agents psychiques qui contribuent à la production du sens ${ }^{10}$.

Le corpus des tatouages est à mon sens une bonne illustration empirique des limites de la conception binaire et anthropocentrée de l'énonciation, et permet de proposer une approche renouvelée du contexte en termes d'environnement et de distribution.

\subsection{Des configurations énonciatives hétérodoxes}

27 Je ne contesterai pas que, comme toute production verbale, le tatouage puisse être lu, quel que soit le mode de lecture et le lecteur. Comme le précise S.-A. Lamer: "Le tatouage et le perçage se situent dans l'ordre du signe, ce qui les place dans une perspective plus large du sens et des valeurs. La visibilité de ce signe l'inscrit d'emblée dans le domaine relationnel. » (1995: 152) On est alors dans un système relationnel, sur un mode particulier, ce que décrit bien F. Baillette :

Toute inscription corporelle a effectivement pour finalité d'être un jour consultée ou questionnée. La marque produit du sens, elle interpelle, désigne, énonce et dénonce. Aussi, qu'elle soit publique ou secrète, mise en évidence ou discrète, la trace déposée doit pouvoir être vue ou simplement entraperçue (voire fantasmée). (Baillette $2003: 62$ )

Que les tatouages écrits soient lisibles n'est pas en question, certains d'entre eux, j'y reviendrai, sont même marqués par la deixis, ce qui les rend hautement " énonciatifs » à la manière standard. Ce qui me semble à réinterroger, ce sont les modalités de la lecture et du déchiffrage, qui ne sont pas aussi évidentes que la théorie standard de l'énonciation le laisse croire, et qui enlèvent à la fonction de communication son évidence (sur ce point voir l'introduction de Banfield 1995 qui fait une fine analyse critique du dogme communicationnel des années 1960 et 1970, et les travaux de G. Philippe qui permettent de relativiser ce qu'il appelle le "tout énonciatif»). Le tatouage est en effet lu de manière particulièrement aléatoire et non prévisible, puisqu'il est souvent caché ou "cachable", ce qui rend son adresse pour le moins indéfinie : l'interlocuteur-lecteur des tatouages scripturaux n'est pas réductible à un " autre » humain pris dans une interaction, mais constitue plutôt un agent de lecture virtuel distribué dans l'environnement cognitif extérieur du tatoué. Cette indéfinition 
touche également l'instance énonciative : on verra que la question du locuteur et de son intention communicative est loin d'être simple dans le cas des écritures corporelles.

J'examine ici successivement trois configurations énonciatives qui brisent selon moi le cadre binaire de l'énonciation standard, puis la configuration particulière du tatouage dissimulé au tatoué lui-même.

\subsection{1. Énoncés prédicatifs en troisième personne}

Une forme très fréquente pour les tatouages écrits est l'énoncé prédicatif en troisième personne sans thème explicite ; autrement dit, une information est donnée à propos d'une entité qui n'est pas explicitée, mais que l'on infère de la situation: le thème semble être le porteur du tatouage, puisque le prédicat est supporté par son corps. "Semble ", car l'énoncé ne contient pas de marques indicatives sur l'identification du support de l'information. C'est particulièrement le cas pour les tatouages identitaires exhibés sur les parties visibles du corps, face (front, joue, cou), bras et avant-bras, mains, jambes, chevilles, dos. Enfant du malheur, Martyr militaire, Esclave du PCUS (tatouage de goulag) MS 13 (nom d'un gang hispanique de Los Angeles, décrit par Mohamed Habibi qui publie des photos de tatoués sur son blogue kutub.over-blog.com, dans la rubrique "Gangs à fleur de peau»), autant de dénominations qui sont des catégorisations de soi par un procédé proche de l'étiquetage. On mettra également dans cette catégorie certains tatouages de resignification ${ }^{11}:$ KKK sur la main d'un GI noir, bitch sur la hanche d'une femme (www.fotosearch.fr).

31 Cette lecture est situationnellement cohérente mais linguistiquement invérifiable : qui me dit que l'homme qui porte le tatouage Enfant du malheur s'autodésigne? Qui me dit que le front tatoué du sigle MS accompagné d'un 13 sur le menton appartiennent à un membre du gang? et que la femme qui porte bitch sur la hanche, l'homme qui porte Insane sur le bras, s'autodéfinissent, même ironiquement? En l'absence de marques langagières, on ne peut répondre à cette question qu'intuitivement, ou en faisant appel à des données non linguistiques: il est très difficile d'évaluer le consentement du tatoué, et donc sa responsabilité énonciative. Si les tatouages de prisonniers et de militaires, parce qu'ils sont des plaintes et des énoncés contestataires, semblent bien relever d'un automarquage (au sens où le tatoué demande l'inscription), les tatouages d'affiliation (ceux des gangs ou de certains groupes militaires restreints comme les légionnaires) sont plus douteux : comme l'explique J.-É. Lundy à propos de la Légion étrangère, les tatouages résultent d'une contrainte narcissique, c'est-à-dire de l'imposition d'images du moi conformes à l'institution et nécessaires pour être reconnu par elle et par les autres membres ${ }^{12}$. Les tatouages matérialiseraient alors ce lien imaginaire faisant se rapprocher le moi du légionnaire de l'idéal de l'institution de la Légion Étrangère en garantissant ce que le médecin appelle un "contrat narcissique " (Lundy 1989 ; fig. 3). Toutes proportions gardées, c'est un phénomène analogue qui se produit dans le cas des tatouages de soumission dans le cadre des contrats sadomasochistes: le site jeune-soumise.com présente une jeune femme tatouée sur une fesse Propriété de maitre Nathan. Automarquage, hétéromarquage non consenti ou catégorie intermédiaire indécidable? 

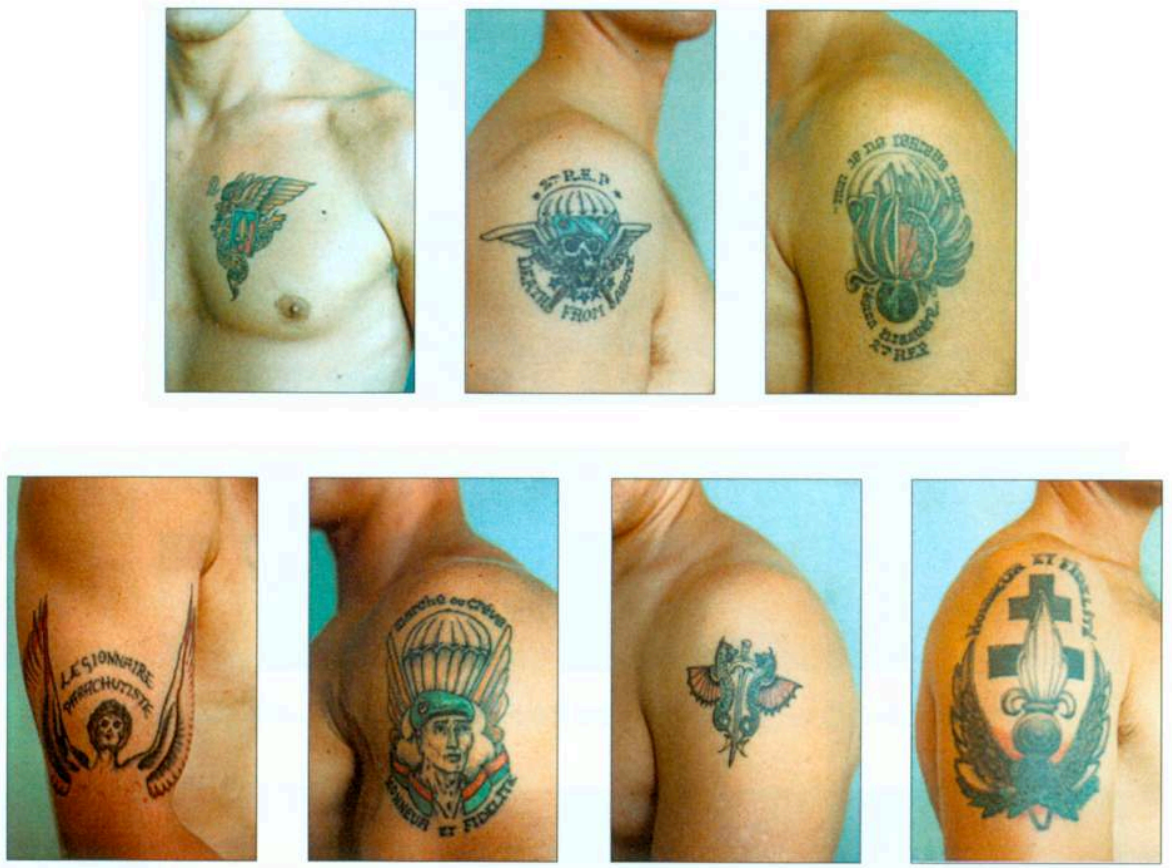

Jean-Éric Lundy, Rapports du tatouage au Contrat Narcissique dans la Légion étrangère, mémoire pour le CES de psychiatrie, université René-Descartes, Cochin-Port-Royal, planche III, non paginé, 1989.

On peut en revanche être sûr de l'hétéromarquage non consenti pour les tatouages appliqués sur les vaincus pendant certaines guerres, pour les immatriculations des déportés pendant la Seconde Guerre mondiale (tatouages de désidentification plutôt que d'identité) et pour d'autres marques forcées dont la pratique n'a pas disparu (voir plus haut le cas des initiales des proxénètes tatouées sur le cou de leurs prostituées) ${ }^{13}$.

Dans tous ces cas, le tatouage pose un problème évident de présence et d'identification $\mathrm{du}$ locuteur, que l'on ne peut approcher me semble-t-il qu'en modifiant le cadre théorique de l'énonciation, et en postulant une nature non communicationnelle de certains énoncés (voir 2.3.).

\subsection{2. Énoncés déictiques en première personne}

Deuxième configuration énonciative, les énoncés en je, qui semblent entrer dans le cadre canonique du "discours» benvenistien, puisqu'ils présentent une deixis personnelle explicite. Ils posent cependant des problèmes d'adresse et de lecture : à qui sont-ils adressés ? quel est leur lecteur-interpréteur? Les exemples suivants montrent qu'il n'est guère possible de postuler une situation de communication traditionnelle, et que l'adresse doit être repensée de manière distribuée, c'est-à-dire sans identification précise d'un récepteur mais dans l'idée, un peu sur le modèle de la «bouteille à la mer », d'une réception aléatoire, multiple et anonyme ${ }^{14}$, accomplie par l'environnement tout entier :

- Je suis cochon au lit (passim dans le corpus)

- J'ai aimé j'ai souffert maintenant je hais (Delarue, Giraud 1950, planche 15 avec

dessins de têtes de femmes et scènes de baisers)

- J'ai souri j'ai souffert (à la base du cou, Pierrat, Guillon, 2004 : 53) 
- Mon rêve est de revoir ma mère (sur le dos, Pierrat, Guillon 2004 : 96)

- Ma haine aux gradés (Tagnaouti 1979)

- Marine, je suis venu à toi sans te connaitre, je te servirai sans t'aimer, je te quitterai sans regret (Tagnaouti 1979)

- La Marine sera mon tombeau (Tagnaouti 1979)

- Je remercie mes maitres communistes pour cette jeunesse heureuse (tatouage de goulag, passim dans le corpus)

- Le passé m'a trompé, le présent me tourmente et l'avenir m'épouvante (fonds Lacassagne) virtuel doit se référer à des connaissances mémorielles et culturelles pour construire presque ex nihilo le contexte qui lui permettra l'interprétation de l'énoncé. Dans cette construction, les éléments matériels jouent un rôle important: corps biologique du tatoué comprenant d'autres marques corporelles éventuelles, vêtements, environnement géographique, etc. C'est en cela que l'énoncé tatoué mobilise un environnement cognitif : les données contextuelles résultent de la cognition des agents qui font appel à l'ensemble des données matérielles et immatérielles pertinentes pour la lecture de l'énoncé.

\subsubsection{Adresses en deuxième personne}

Dernier dispositif en jeu dans le corpus: les énoncés en deuxième personne, qui présentent donc une adresse explicite et qui semblent impliquer naturellement une interprétation communicationnelle. Là encore, le dispositif énonciatif est élargi puisque le « récepteur » n'existe pas dans le cadre d'une situation de communication inexistante, mais reste virtuel. On peut dire qu'il est distribué dans l'environnement, matérialisé dès qu'un œil, au sens d'instance socialement construite, quel qu'il soit, le capte. On n'est pas dans une situation d'interaction, dans la mesure où une relation verbale avec l'autre n'est pas construite. Seule demeure la production d'une adresse de la part du tatoué (je n'ai trouvé aucun tatouage non consenti en deuxième personne, ce qui est notable), adresse non adressante, si l'on peut dire : lise qui passera, comprenne qui pourra, reçoive qui voudra.

l'autre qui établissent une relation visuelle entre le tatoué et le regard qui le saisit :

- Vous qui me regardez, j'ai été une victime de l'armée (bas du dos, Delarue, Giraud $1950: 41$ )

- Oh! M...! encore un c... qui me regarde [bras gauche]; As-tu un louis à mettre

dans le commerce, oui ou non? [avant-bras gauche] (Delarue, Giraud $1950: 46$ )

- Regarde Saint Christophe puis va-t-en rassuré (Schiffmacher 2005 : 340)

Ces énoncés sont intéressants dans la mesure où ils semblent confirmer l'hypothèse non communicationnelle: le tatouage n'engage pas la conversation, mais mobilise le regard, à la manière d'une sémiotique non verbale.

Seconde catégorie bien repérable, celle des impératifs, contenant une sous-catégorie qu'on peut appeler du « memento » (deux derniers exemples) :

- Souffre mais tais-toi (Delarue, Giraud 1950, Planche 66)

- Ouvre 1[œil dessiné] le bon (Delarue, Giraud 1950, Planche 67)

- Souffre en silence (ventre, Pierrat, Guillon 2004 : 38)

- Nosce te ipsum (haut du torse, Lautman 1994, p. 53) 
- Stay calm (Saltz $2006: 167)$

- Stay true (www.matton.fr)

- Souviens-toi (milieu du torse avec des chaînes, Pierrat, Guillon 2004 : 55)

- Remember (base du cou Pierrat, Guillon 2004 : 60)

41 La question de l'adresse se pose ici de manière évidente : s'il faut bien postuler un énonciataire puisque les énoncés contiennent un marqueur langagier explicite (la flexion du verbe en personne), nul ne peut dire quelle est l'instance désignée, qui constitue une forme virtuelle non contextualisée et donc indéfinie. Ces énoncés en deuxième personne ont une valeur gnomique, ressortissant plus à la sagesse qu'à l'adresse, ce qui est cohérent avec l'absence de coordonnées spatiales et temporelles : le tatouage étant une inscription permanente mais mobile, il ne permet pas l'installation d'une situation d'énonciation socialement déterminée. Sa configuration énonciative est de l'ordre de l'imaginaire.

\subsubsection{Le cas des énoncés cachés}

42 La visibilité des tatouages est loin d'être une évidence, y compris chez les tatoueurs. Bruno, tatoueur à Pigalle (auteur de Tatoués, qui êtes-vous ?), refuse de tatouer le visage et les mains (Le Cornec 1984 : 94). Le visage tatoué est, au mieux une originalité que seule une star comme Mike Tyson peut se permettre (tatouage tribal de style maori autour de l'œil), au pire un « suicide social », comme le précise le guide Tout savoir sur le tatouage rédigé par l'équipe de Tatouage magazine (p.75), propos confirmé par les tatoueurs du plus ancien tattoo shop d'Allemagne: "Nous ne voulons pas que les gens rencontrent des difficultés dans leur vie professionnelle» (Vandekerchove 2005 : 69). Une des attitudes normatives par rapport au tatouage semble être une visibilité contrôlée : il faut pouvoir le montrer quand on le souhaite.

Dans le corpus que j'ai constitué, la dissimulation correspond à l'un des trois dispositifs suivants :

- les tatouages sont dissimulés par les vêtements en situation sociale et ne se découvrent que dans la nudité de la ou des zone(s) concernée(s) ;

- ils sont appliqués à l'intérieur du corps (mes exemples concernent la bouche) ;

- ils sont dissimulés au regard du tatoué mais visibles aux regards extérieurs.

La première configuration est très fréquente et correspond au discours de nombreux tatoués. Dans son mémoire sur les tatouages, S. Dupont recueille des témoignages de ce type chez ses enquêtés, par exemple Jean :

Je veux pas qu'il puisse se voir en position, je veux dire, on va dire quand je suis habillé, normalement, en jean T-shirt je veux pas que ça se voie

Je veux pas que ce soit quelque chose d'ostentatoire, c'est pour moi que je le fais,

c'est pas pour les autres, quoi, euh on va dire c'est pour mon bien-être

[...] le côté quand même un peu ça se voit sans se voir c'est quand même sympa

(Dupont 2006)

Et de fait, hanches, fesses, poitrine ou ventre sont des zones fréquentes de tatouage.

La deuxième configuration est la plus rare, mon corpus ne comprenant que trois tatouages à l'intérieur du corps : il s'agit de personnes qui portent respectivement les mots alien, pork! et pony sur la face interne de la lèvre inférieure, dans le sens de la lecture pour un regard extérieur (recueillis dans Saltz $2006: 80,81$ et 101). La porteuse d'alien explique : "It's an unseen place, and represents a feeling of separateness, of not belonging ${ }^{15}$. » Le second est cuisinier dans un restaurant d'entreprise et il explique son 
tattoo pork! par un lien avec son métier, retrouvant la vieille tradition du tatouage professionnel. La troisième est chanteuse d'opéra : «I'm an opera singer so I couldn't have a tattoo in a compromising place. My nickname is Pony ; it is the name of my former band; also my dad is a horse trainer, and inside the lip is where you tattoo horses to ID them ${ }^{16}$.» (Saltz 2006: 101; fig. 4) Ces trois cas sont très difficiles à interpréter sur le plan énonciatif, même avec l'intéressante explication zoocentriste de Pony, mais semblent confirmer la nature non communicationnelle de l'écrit tatoué.

Figure 4

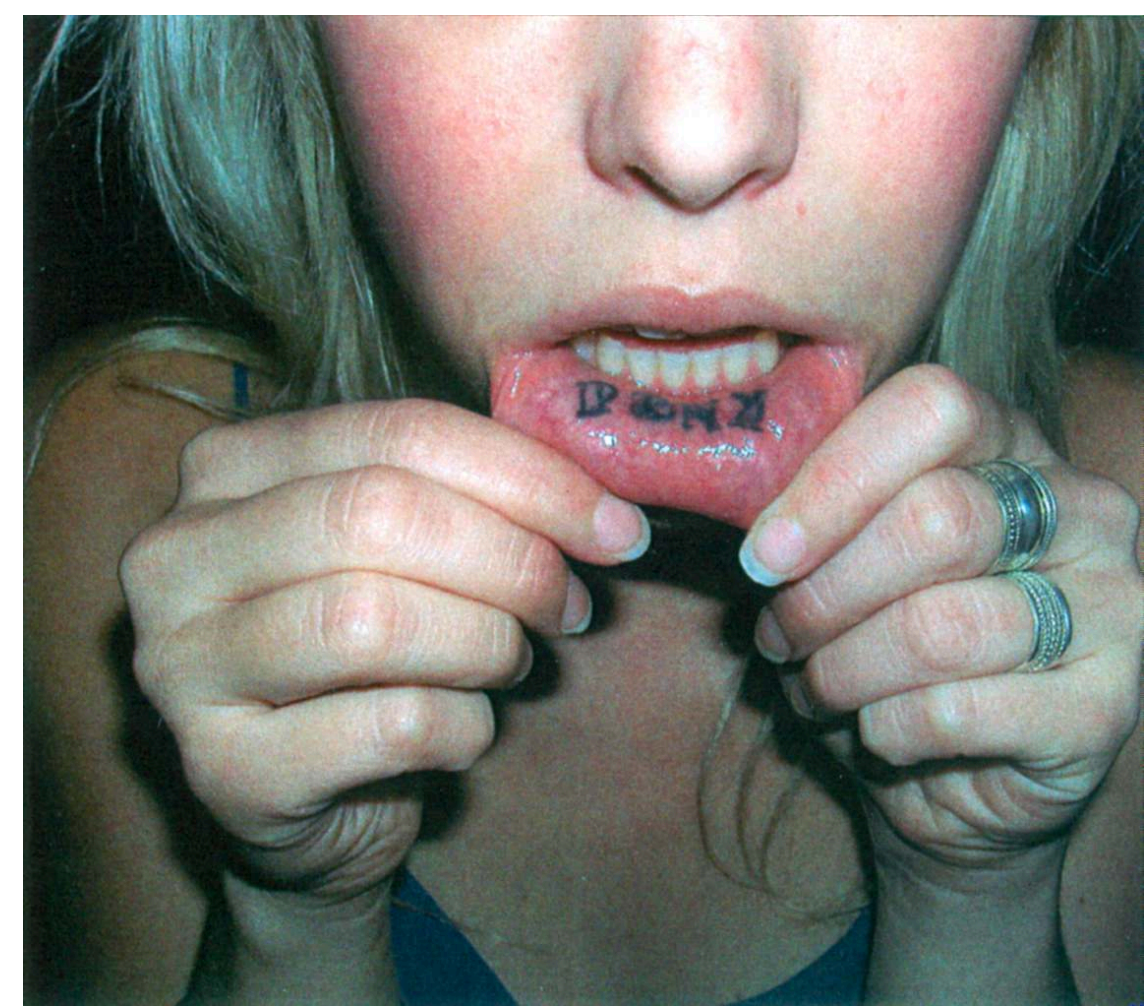

Ina Saltz, Body Types. Intimate Messages Etched in Flesh, New York, HNA, 2006, p. 101.

La troisième configuration est aussi fréquente qu'étonnante sur le plan énonciatif : les tatouages non visibles par les tatoués. Ce sont essentiellement les marques inscrites dans le dos, sur les fesses ou sur l'arrière des cuisses. On citera entre mille les tatouages de M. Materazzi (l'insulteur de Z. Zidane lors de la coupe du Monde de 2006) qui porte son prénom et celui de sa femme le long de la colonne vertébrale (Match 2006 : 47), celui de cette jeune femme qui arbore Poison free en haut du dos, en hommage à sa sortie de la toxicomanie (Saltz 2006 : 151, chapitre 8 sur les « Belief Systems ») ou encore cet Only God can judge me (avec une variante latine Deus solus me judicat dans Saltz 2006 : 133), qui laisse le linguiste énonciativiste un peu perplexe. En effet, comment interpréter le fait que le porteur d'une inscription ne puisse pas la lire (sauf dans un miroir et donc à l'envers) ? Ce qui amène une première réponse : l'énonciateur de l'inscription n'est pas son porteur, réponse qui ne peut être faite cependant que si la théorie standard prévoit que l'énonciateur puisse être son propre énonciataire. Autrement dit : dans la théorie standard, l'énonciateur a-t-il accès à l'énoncé qu'il produit? Il ne semble pas que ce point soit traité dans la littérature sur la question, donc on peut supposer qu'il peut y avoir accès ou non. Il n'y a donc pas de raison de postuler une distinction entre le 
porteur du tatouage et son énonciateur, ce qui amène encore un peu d'eau au moulin hétérodoxe : si l'énonciateur ne peut lire l'énoncé qu'il produit, alors la fonction de communication de l'énoncé en question est plus que douteuse et une situation d'interaction n'est guère envisageable. Il faut peut-être voir les choses autrement, et décrire l'énonciation comme une distribution des informations élaborées dans l'environnement cognitif et non pas « contenues » dans un message.

48 Je termine par un essai de synthèse qui rassemble les données énonciatives « hétérodoxes » recueillies au long du travail.

\section{3. Éléments non standard de théorie énonciative}

Dans La Raison, le langage et les normes, S. Auroux signale qu'en linguistique, une évolution ou une modification des exemples est l'indice sûr d'un changement de théorie (Auroux 1998: 192 ; passage en exergue de l'article). Je pense que les tatouages ont ce rôle dans la théorie de l'énonciation. Les tatouages scripturaux constituent en effet un ensemble d'énoncés dont les caractéristiques impliquent une position antiindexicale quant à certains types d'écrits, et qui permettent de défendre, à la suite d'A. Banfield, l'idée que le langage n'est pas toujours communicationnel: certains énoncés, dont les tatouages, n'entrent pas dans le dispositif standard qui fonde toute énonciation sur une interaction communicationnelle explicite ou implicite, de surface ou de manière primitive. Dire, ce n'est pas forcément dire à quelqu'un, ce peut être dire tout court, dire pour soi ou simplement exprimer, marquer ou formuler son expérience, installer un lien avec le réel. Ma position implique corrélativement, on le verra, de plaider pour un certain référentialisme.

\subsubsection{La fonction « bio » de la corpographèse (1) : autobiographie}

50 J'ai montré que la situation énonciative du tatouage ne correspondait pas au dispositif canonique issu des propositions d'É. Benveniste et reconduit par les linguistiques interactionnelles et dialogiques: absence de marques indexicales dans les énoncés (énoncés en troisième personne), absence d'énonciataire identifiable (énoncés en deuxième personne) et parfois même absence d'énonciateur, dans le cas des énoncés non accessibles à leur porteur, qu'il soit désigné comme énonciateur ou non.

51 Les tatouages sont alors un peu ces « phrases sans parole » décrites par A. Banfield dans le champ littéraire ${ }^{17}$, qui n'ont pas de fonction communicationnelle déclarée. Mais quelles fonctions leur attribuer? Des fonctions qui mettent le corps vivant au centre du processus d'écriture, l'autobiographie et la construction de la biosubjectivité.

52 Tous les témoignages des tatoués insistent sur la dimension autobiographique des inscriptions corporelles, rejoignant par là les fonctions des marques tégumentaires dans les civilisations primitives et antiques. Les enquêtés de S. Dupont ne font pas exception à la règle :

$53-$ Jean : Je le vois comme une sorte de sortie de ce que j'étais avant.

- Cécile : Et puis en fait on avait décidé de se marier très peu de temps avant et euh on s'était dit qu'en fait bah c'était peut-être le moment de se faire le tatouage avant le mariage. (Dupont 2006)

54 «Le tatouage avant le mariage »: sous la rime spontanée, la fonction initiatique, la scansion des âges de la vie humaine (dans certaines civilisations, on tatouait les femmes 
ayant eu leur premier rapport sexuel). Les tatouages apparaissent alors comme des autobiographèmes, sous de nombreuses variantes mais avec ce point commun du discours «sur soi pour soi » qu'illustrent bien les inscriptions dans la bouche mentionnées plus haut, ainsi que les nombreuses dates de naissance et nationalités que l'on rencontre souvent (par exemple : made in Canada).

Il faut corrélativement souligner la forte dimension mémorielle du tatouage, sorte de lieu de mémoire épidermique qui conserve le souvenir des êtres aimés, des évènements de la vie (puberté, maternité, mariage, etc.), des batailles livrées (pour certains soldats, un corps-drapeau s'orne du nom des victoires), des épreuves endurées (le tatouage concentrationnaire, à vocation "pratique " pour le tatoueur, a été doté par l'histoire d'une fonction mémorielle, parfois militante, pour le tatoué), mais aussi de détails pratiques comme le groupe sanguin (habitude des SS et encore de certaines armées contemporaines). À cet égard, la démocratisation récente du tatouage dans nos sociétés est sans doute un indicateur non négligeable de la construction des identités et de la gestion des mémoires personnelles et collectives.

\subsubsection{La fonction « bio » de la corpographèse (2) : biosubjectivité}

Avec l'idée d'une "biosubjectivité » (le concept est de B. Andrieu), on peut expliquer que les tatouages ne reposent pas sur la communication mais sur la construction corporelle, c'est-à-dire la modification du sujet par un «design biosubjectif de la matière " :

Être un corps naturel est désormais insuffisant pour être humain. L'identité singulière du corps reçu par la nature fournit dans sa matière des possibilités de normativité nouvelle. Devenir soi-même exige plus qu'une simple transformation $\mathrm{du}$ corps naturel. La manière d'être ne traduit qu'un contrôle de l'apparence corporelle, tandis que la matière d'être résoudrait l'opposition entre objet-sujet en matérialisant la forme choisie par le sujet pour se définir. Le corps humain n'est pas seulement biologique car il produit dans la culture des normes adaptées au vécu de son vivant. (Andrieu 2004a : 342)

Le tatouage comme "matière d'être " permettrait alors un devenir-sujet par le corps, au moyen d'une nouvelle scripturalité qui inaugurerait un autre usage du langage : une corpographèse qui produit le sens du sujet, et non de l'énoncé, et où l'interaction se fait entre l'homme et son environnement, et non forcément entre deux instances de discours. "L'homme, signale S. Auroux, est avant tout une structure biologique (un corps) qui interagit avec un environnement et d'autres corps. Le phénomène fondamental est la constitution d'outils, ce qui implique l'instrumentalisation autant de l'environnement que du corps propre» (Auroux 1998: 7). En ce sens le tatouage est étroitement dépendant d'un environnement cognitif (au sens culturel du terme) qui permet au sujet la co-construction de cette nouvelle (bio)subjectivité.

\subsubsection{Unspeakable Tattoos : un bioréférentialisme?}

L'hypothèse de la corpographie non communicationnelle implique, rappelons-le, qu'il n'y ait pas forcément de lecteur, donc pas de retour interprétatif, ni co-énonciation active. Désénoncé, le tatouage échappe, dans une certaine mesure, à la subjectivité du langage et acquiert de ce fait une puissance, non pas objective, mais référentielle (pour une synthèse complète et problématisée des questions liées à l'objectivisme et au référentialisme, et pour une position référentialiste fine en linguistique, voir Achard- 
Bayle 2007). Il me semble en effet que la position «hyper-indexicale ( (toute forme de langage est communicationnelle et toute production verbale s'intègre dans un échange intersubjectif), comme celle d'ailleurs qui plaide pour la co-construction de l'échange verbal, a tendance à minorer la fonction référentielle du langage : par hypostasie de la fonction énonciative, on oublie la référence ${ }^{18}$. Or la corpographie implique une ligne de continuité entre les fonctions pensantes et parlantes de l'esprit et la réalité du corps et de l'environnement extérieur. En ce sens, il est un «instrument ", pour reprendre le vocabulaire de S.Auroux, de négociation du rapport du sujet au réel biologique, matériel, social et culturel ${ }^{19}$.

\section{BIBLIOGRAPHIE}

N. B. : certains ouvrages constituant à la fois des ouvrages de référence et des corpus d'étude sont présents dans les deux sections.

Achard-Bayle, G., 2007, « Les réalités conceptuelles et leur ancrage matériel. Les sémantiques cognitives et la question de l'objectivisme », numéro spécial de la revue électronique Corela (Cognition, Représentation, Langage), « Contextes, discours, cognitions », en ligne sur http:// corela.edel.univ-poitiers.fr/index.php?id=1532.

Andrieu, B., 2003, Le Somaphore. Naissance du sujet biotechnologique, Liège, Sils Maria.

-, 2004a, «La santé biotechnologique du corps sujet », Revue Philosophique de France et de l'Étranger, «La biologie et ses questionnements philosophiques au début du XIX siècle », Paris, PUF, p. 339-344.

,- 2004 b, « Somaphore et corps biosubjectif », Multitudes 14, «Philosophie de la biologie », p. 59-69.

Anzieu, D., 1995 [1985], Le Moi-peau, Paris, Dunod.

Assouline, P., 2005, « Marqués à vie ", Le Monde 2, 8 janvier 2005, p. 17-25.

Auroux, S., 1998, La Raison, le langage et les normes, Paris, PUF.

Baillette, F., 2003, «Inscriptions tégumentaires de la loi », Quasimodo, $n^{\circ} 7$ (« Modifications corporelles »), Montpellier, p. 61-88, disponible en ligne sur http://www.revue-quasimodo.org/ PDFs/7 - Tatouage Loi Inscription.pdf.

Banfield, A., 1995 [1982], Phrases sans parole. Théorie du récit et du style indirect libre, Paris, Le Seuil. Benveniste, É., 1966 [1946], « Structure des relations de personne dans le verbe », dans Problèmes de linguistique générale 1, Paris, Gallimard, p. 225-236.

-, 1966 [1956], « La nature des pronoms », dans Problèmes de linguistique générale, $\mathrm{n}^{\circ}$ 1, Paris, Gallimard, p. 251-257.

-, 1974 [1970], « L'appareil formel de l'énonciation », dans Problèmes de linguistique générale, $\mathrm{n}^{\circ}$ 2, Paris, Gallimard, p. 79-88.

Borel, F., 1992, Le Vêtement incarné. Les métamorphoses du corps, Paris, Calmann-Lévy. 
Delarue, J. et Giraud, R., 1950, Les Tatouages du « Milieu », Paris, La Roulotte, avec portfolio de

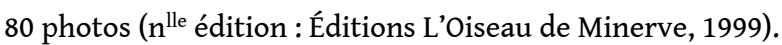

Bruno C, Maitre tatoueur, 1970, Tatoués, qui êtes-vous ?, Bruxelles, Éditions de Feynerolles.

Culioli, A., 1990-2000, Pour une linguistique de l'énonciation, tome $1:$ « Opérations et représentations », 2000 [1990]; t. 2 :« Formalisation et opérations de repérage », 1999a; t. 3 : « Domaine notionnel », 1999b, Paris, Ophrys.

Dupont, S., 2006, Formation de soi et Socialisation. Le tatouage : une forme de biographisation, mémoire de Master 2 en Sciences de l'éducation, université Paris 13. Annexe $3:$ « Entretiens avec des personnes tatouées ", sans pagination.

Gilbert, A.-M., 1970, Propos sur les tatouages, thèse de doctorat en médecine, université de Poitiers, Faculté de médecine et de pharmacie.

Hutchins, E., 1994 [1991], « Comment le cockpit se souvient de ses vitesses » [trad. de « How a Cockpit Remembers its Speed »], Sociologie du travail, $n^{\circ}$ 4, p. 461-473.

Jacques, F., 1985, L'Espace logique de l'interlocution, Dialogiques II, Paris, PUF, coll. « Philosophie d'aujourd'hui ».

Kerbrat-Orecchioni, C, 2006, L'Analyse du discours en interaction, Paris, A. Colin.

Lacassagne, A., 1881, Les Tatouages. Étude anthropologique et médicolégale, Paris, Librairie J.-

B. Baillière et fils.

Lamer, S.-A., 1995, « Graffiti dans la peau. Marquages du corps, identité et rituel », Religiologiques, $\mathrm{n}^{\circ} 12$, p. $149-167$.

Le Blond, A. $\mathrm{D}^{\mathrm{r}}$ et Lucas, A. $\mathrm{D}^{\mathrm{r}}, 1899$, Du tatouage chez les prostituées, Paris, Société d'éditions scientifiques.

Le Breton, D., 1992, La Sociologie du corps, Paris, PUF.

-, 2002, Signes d'identité. Tatouages, piercings et autres marques corporelles, Paris, Métailié.

-, 2003 [1990], Anthropologie du corps et Modernité, Paris, PUF.

Le Cornec, F., 1984, Tatouages et Détatouages. Étude historique, technique et psychosociale, thèse pour le doctorat en médecine, Paris, université Pierre-et-Marie-Curie.

Lundy, J.-É., 1989, Rapports du tatouage au contrat narcissique dans la Légion étrangère, mémoire pour le CES de psychiatrie, université René Descartes, Cochin-Port-Royal.

Marzano, M. (dir.), 2007, Dictionnaire du corps, Paris, PUF coll. « Quadrige ».

Ouellet, P., 1984, «La désénonciation : les instances de la subjectivité dans le discours scientifique ", Protée, nº 12 (2), p. 43-53.

Paveau, M.-A., 2006, Les Prédiscours. Sens, mémoire, cognition, Paris, Presses Sorbonne Nouvelle.

-, 2007a, «Lettres du corps : les tatouages discursifs » communication au colloque Effets de peau. La peau pour le dire, IIIe rencontres de Valvert, centre hospitalier Valvert, Marseille, juin.

,$- 2007 \mathrm{~b}$, « Discours et cognition. Les prédiscours entre cadres internes et environnement extérieur ", numéro spécial de la revue électronique Corela (Cognition, Représentation, langage) : «Contextes, discours, cognitions », http://corela.edel.univ-poitiers.fr/index.php?id=1550.

Philippe, G., 2000, « Présentation », Langue française, $n^{\circ}$ 128, « L'ancrage énonciatif des récits de fiction ", p. 3-8. 
-, 2002, «L'appareil formel de l'effacement énonciatif et la pragmatique des textes sans locuteur ", dans R. Amossy (dir.), Pragmatique et Analyse des textes, Presses Universitaires de TelAviv, p. 17-34.

Pierrat, J., Guillon, É., 2000, Les Hommes illustrés, le tatouage des origines à nos jours, Clichy, Larivière.

-, 2004, Le Tatouage à Biribi. Les vrais, les durs, les tatoués, Paris, Larivière.

Pozzuoli, A., 2005, Tatouages. Une histoire et des histoires, anthologie, Paris, Les Belles Lettres.

Rabatel, A., 2004, «Stratégies d'effacement énonciatif et posture de surénonciation dans le Dictionnaire philosophique de Comte-Sponville », Langages, $\mathrm{n}^{\circ}$ 156, p. 18-33.

Renaut, L., 2004, « Les tatouages d'Ötzi et la petite chirurgie traditionnelle », L'Anthropologie, ${ }^{\circ}$ 108, p. 69-105, disponible en ligne sur http://www.sciencedirect.com/science/article/pii/ S0003552103000840.

Schegloff, E., 1992, « Rethinking Context: Language as an Interactive Phenomenon », dans A. Duranti et C. Goodwin (dir.), Another context, Cambridge, Cambridge University Press, p. 191-227.

Tagnaouti, B., 1979, Tatouages en milieu militaire, thèse pour le doctorat de médecine, université de Bordeaux-II.

Tatouage magazine, 2005, « Tout savoir sur le tatouage », Paris, Larivière.

Tenenhaus, H., 1995, Le Tatouage à l'adolescence. De la représentation graphique à la représentation psychique : le corps médiateur, Paris, Bayard.

Vandekerchove, L., 2005 [2002], Le Tatouage. Sociogenèse des normes esthétiques, trad. du néerd. J. Poulin, Louvain-La-Neuve, Academia Bruylant.

Vion, R., 2001, « "Effacement énonciatif” et stratégies discursives », dans A. Joly et M. Mattia (de) (éds.), De la syntaxe à la narratologie énonciative, Paris, Ophrys, p. 331-354.

\section{Corpus}

\section{Recueils imprimés}

Album d'un tatoeur [sic] lyonnais en 1889, recueil de dessins, avec au moins deux mains différentes, $\mathrm{D}^{\mathrm{r}}$ Batut médecin major de $2^{\mathrm{e}}$ classe, répétiteur à l'école du service de santé militaire de Lyon. Don au laboratoire de médecine légale de la faculté, 13 février 1897 (fonds Lacassagne, bibliothèque municipale de Lyon).

Grognard, C. (textes) et Lazi, C. (photos), 1992, Tatouage. Tags à l'âme, Paris, Syros alternatives. Keith. Culture et Contre-culture, 2007, magazine gratuit, $n^{\circ}$ 03, dossier « Crazy tattoos », Paris, Who is Keith?

Lautman, V., 1994, Tatouage, New York, Paris et Londres, Abbeville, photos de Vicki Berndt.

Lundy, J.-E., 1989, Rapports du tatouage au contrat narcissique dans la Légion étrangère, mémoire pour le CES de psychiatrie, université René-Descartes, Cochin-Port-Royal.

Match, « Materazzi le provocateur », 20-26 juillet 2006, p. 46-49.

Saltz, L, 2006, Body Types. Intimate Messages Etched in Flesh, New York, HNA.

Schiffmacher, H. (dir.), 2005, 1000 Tattoos, Köln, Taschen. 
- et Riemschneider B. (dir.), 2001, Tattoos, Köln, Taschen.

Tattoo style, oct.-déc. 2006, Mannheim, Huber Verlag.

\section{Sites et banques d'images en ligne (consultés en juillet 2008)}

http://pro.corbis.com (banque d'images).

http://www.fotosearch.fr (banque d'images).

http://kutub.over-blog.com (archives du blogue de Mohamed Habibi fermé en 2005 rubrique

« Gangs à fleur de peau »).

http://www.matton.fr (banque d'images).

http://www.tattoos.com (portail du tatouage).

http://www.corpsenfolie.com, rubrique écritures and lettring (site sur les marquages corporels).

\section{NOTES}

2. H. Tenenhaus précise que les tatouages «scripturaires" représentent environ le tiers des tatouages examinés dans son enquête sur les adolescents (Tenenhaus 1993 : 166). Les écrits sont en effet plus nombreux dans les tatouages qu'il n'y parait au premier abord, beaucoup d'entre eux étant verbo-iconiques.

3. Sur ces questions voir Le Breton 1992.

4. Les illustrations de l'article figurent dans le cahier photographique en fin de volume.

5. J. Delarue et R. Giraud présentent le cas amusant d'une série de quatre prénoms de femmes tatoués sur un bras dont deux barrés (1950: 32). I. Saltz montre un tatouage d'hommage énumérant la liste des astronautes de la NASA (2006: 177). Le ou la tatoué(e) porte volontiers le prénom de ses enfants, plus stable que celui de son ou sa conjoint(e).

6. Les exemples sont tirés de Lacassagne 1881.

7. Édouard Kouznetsov, 1974, Journal d'un condamné à mort, Paris, Gallimard, « Témoins ", [1 $1^{\text {re }}$ édition, sous le titre Dnevniki en 1973 aux Éditeurs Réunis], p. 196-200 [Note de Baillette].

8. Il faut cependant préciser que pour P. Ouellet, la désénonciation des textes scientifiques n'est que de surface, le dispositif interactionnel étant conservé, comme on peut s'y attendre en 1984, époque où triomphe la théorie de l'énonciation réactivée par les traductions de Bakhtine en français : «Dans le discours scientifique, la plupart, sinon la totalité, des présuppositions ou des proto-énoncés transformés renvoie (sic) à une instance subjective et agentive, animée et anthropomorphe, que les multiples transformations des "relations primitives", structurant les états de faits en "relations prédicatives" constitutives de l'énoncé contribuent à masquer, cacher, effacer. » (Ouellet $1984: 44$ )

9. Pour R. Vion, l'effacement énonciatif est une "stratégie, pas nécessairement consciente, permettant au locuteur de donner l'impression qu'il se retire de l'énonciation, qu'il "objectivise" son discours en "gommant" non seulement les marques les plus manifestes de sa présence (les embrayeurs) mais également le marquage de toute source énonciative identifiable » (Vion 2001 : 334 ; je souligne).

10. Je fais référence ici aux acquis de la cognition distribuée, l'un des sous-courants de la cognition sociale américaine, alternative à la cognition internaliste traditionnelle, dont l'un des promoteurs est E. Hutchins. Dans une étude célèbre sur l'élaboration de l'information à bord d'un avion, il montre que l'information n'est pas échangée entre les deux individus aux commandes, 
mais distribuée entre des agents psychiques humains (pilote et copilote) et non humains (instruments de mesure, notes, listes, etc.) ; voir Hutchins 1994.

11. J'appelle resignification à la suite de $\mathrm{J}$. Butler le phénomène qui consiste à inverser l'usage stigmatisant ou insultant d'une dénomination pour en faire un étendard identitaire : par exemple nègre dans le contexte de la décolonisation, Chiennes de garde comme nom de groupe féministe, salopes dans le « Manifeste de 343 salopes » plaidant pour l'avortement, ou plus récemment queer comme désignation d'une identité sexuelle alternative à l'hétéronormativité et d'un courant de recherches (sur la resignification, également appelée remise en scène [restaging] voir Butler 2004 [1997] : 38-41).

12. Cette fonction du tatouage est sans doute à rapprocher de celle qu'il assume dans les sociétés traditionnelles, marquant les changements d'âge et d'état. On ne peut qu'y associer le fonctionnement du nom propre dans ces mêmes sociétés : l'anthroponyme se modifie en effet de la même manière, mais sur décision d'autrui, jamais de soi. Tatouage et nom propre ont en commun le fait de répondre à un besoin d'étiquetage (je remercie Guy Achard-Bayle d'avoir attiré mon attention sur ce point, ainsi que sur l'analogie présentée à la note 14).

13. La thèse de F. Le Cornec mentionne à plusieurs reprises, sur des femmes uniquement, des tatouages non consentis pendant des comas par exemple. Elle ne donne aucun détail, mais ces exemples montrent, d'une part que la pratique du marquage forcé est encore effective, et pas seulement dans des contextes totalitaires ou criminels, et d'autre part, et par conséquent, que la question de l'origine énonciative se pose bel et bien, et de manière cruelle dans ce cas (Le Cornec 1984).

14. Ce type de réception est également, toutes proportions gardées, celle du texte littéraire, selon P. Ricœur par exemple, pour lequel la littérature est l'équivalent d'un «Umwelt », c'est-à-dire l'ensemble de tous les possibles projetables par quiconque sur le texte littéraire. Le récepteur serait alors une virtualité, et on s'éloigne bien du modèle énonciatif standard.

15. «C'est un endroit qu'on ne voit jamais, et ça représente un sentiment de séparation, de désappartenance. »

16. «Je suis chanteuse d'opéra et je ne pourrais donc pas porter un tatouage dans un endroit compromettant. Mon surnom est Pony ; c'est le nom du groupe auquel j'appartenais ; et puis mon père est entraineur de chevaux et c'est à l'intérieur de la lèvre que l'on tatoue les chevaux pour les identifier.»

17. Les énoncés énonciativement désinscrits pour A. Banfield sont les récits fictionnels en troisième personne et ceux au discours indirect libre qui lui semblent ne rendre compte que de l'intériorité mentale du personnage et non pas de sa présence énonciative.

18. C'est aussi la position de G. Philippe à propos du récit littéraire (Philippe 2002).

19. Merci à Guy Achard-Bayle, Sémir Badir et Pierre Zoberman de leurs suggestions et discussions qui ont enrichi et affiné mon approche dans ce travail.

\section{RÉSUMÉS}

Les tatouages scripturaux semblent rendre crédible l'idée selon laquelle le corps humain est le support d'une production discursive dans la manière traditionnelle de la communication indexicale, caractérisée par l'existence de deux locuteurs, une forme linguistique et son interprétation. En effet, l'énonciation tatouée est une forme fort particulière d'énonciation qui fournit plutôt de solides arguments en faveur d'un type non communicatif d'énonciation : les 
locuteurs sont déconnectés et même absents de l'interaction; ils sont aussi la plupart du temps anonymes ; les textes sont parfois dissimulés et donnent donc naissance à des phrases indicibles. Par conséquent, la théorie standard de l'énonciation doit être révisée et on doit proposer d'autres hypothèses, comme la biosubjectivité et le bioréférentialisme.

Scriptural tattoos seem to give credence to the idea that the human body is a support for discourse production in the traditional way of indexical communication, that is, the existence of two speakers, a linguistic form and its interpretation. In effect, tattooed enunciation is a very peculiar one and provides, rather, strong arguments in favor of a noncommunicative type of enunciation: speakers are disconnected and even absent from interaction; they are also most of the time anonymous; texts are sometimes hidden and therefore set up unspeakable sentences. Consequently the standard enunciation theory must be revisited and one has to propose other hypotheses, such as biosubjectivity and bioreferentialism.

INDEX

Mots-clés : environnement cognitif, indexicalité, énonciation non communicationnelle, référentialisme, tatouages scripturaux

Keywords : cognitive environment, indexicality, noncommunicative enunciation, referentialism, scriptural tattoos

\section{AUTEUR}

\section{MARIE-ANNE PAVEAU}

Université Paris 13, CENEL 